\title{
The effect of surface heterogeneity on cloud absorption estimates
}

\section{Article}

Published Version

Chiu, J. C., Marshak, A. and Wiscombe, W. (2004) The effect of surface heterogeneity on cloud absorption estimates. Geophysical Research Letters, 31 (15). L15105. ISSN 00948276 doi: https://doi.org/10.1029/2004GL020104 Available at https://centaur.reading.ac.uk/16772/

It is advisable to refer to the publisher's version if you intend to cite from the work. See Guidance on citing.

To link to this article DOI: http://dx.doi.org/10.1029/2004GL020104

Publisher: American Geophysical Union

All outputs in CentAUR are protected by Intellectual Property Rights law, including copyright law. Copyright and IPR is retained by the creators or other copyright holders. Terms and conditions for use of this material are defined in the End User Agreement.

\section{www.reading.ac.uk/centaur}

\section{CentAUR}

Central Archive at the University of Reading

Reading's research outputs online 


\title{
The effect of surface heterogeneity on cloud absorption estimates
}

\author{
J.-Y. C. Chiu \\ Joint Center for Earth Systems Technology, University of Maryland Baltimore County (UMBC), Baltimore, Maryland, USA
}

\author{
A. Marshak and W. J. Wiscombe \\ Climate and Radiation Branch, NASA Goddard Space Flight Center, Greenbelt, Maryland, USA
}

Received 29 March 2004; accepted 8 July 2004; published 6 August 2004.

[1] This study presents a systematic and quantitative analysis of the effect of inhomogeneous surface albedo on shortwave cloud absorption estimates. We used 3D radiative transfer modeling over a checkerboard surface albedo to calculate cloud absorption. We have found that accounting for surface heterogeneity enhances cloud absorption. However, the enhancement is not sufficient to explain the reported difference between measured and modeled cloud absorption. INDEX TERMS: 0320 Atmospheric Composition and Structure: Cloud physics and chemistry; 1640 Global Change: Remote sensing; 3322 Meteorology and Atmospheric Dynamics: Land/atmosphere interactions; 3359 Meteorology and Atmospheric Dynamics: Radiative processes; 3360 Meteorology and Atmospheric Dynamics: Remote sensing. Citation: Chiu, J.-Y. C., A. Marshak, and W. J. Wiscombe (2004), The effect of surface heterogeneity on cloud absorption estimates, Geophys. Res. Lett., 31, L15105, doi:10.1029/2004GL020104.

\section{Introduction}

[2] Anomalous shortwave cloud absorption is defined as the difference between measured and model-calculated absorption. Regardless of the recent debates about the size of the effect [Ackerman et al., 2003; O'Hirok and Gautier, 2003; Valero et al., 2003], there is no doubt that some discrepancies exist between observed and calculated cloud absorption, which tend to be a bias rather than a random error [Valero et al., 2003]. This excess absorption is on the order of $10 \mathrm{~W} / \mathrm{m}^{2}$ [O'Hirok and Gautier, 2003; Valero et al., 2003]. Any such bias is of concern since radiative transfer models are tacitly assumed to be unbiased in climate modeling and remote sensing applications.

[3] Numerous efforts have been made to identify potential sources of this shortwave cloud absorption bias, including sampling issues in the observations, measurement uncertainties, cloud inhomogeneity, microphysics optical properties, and aerosol loadings [Barker, 1992; Marshak et al., 1997; Valero et al., 1997; Cess et al., 1999; Knyazikhin et al., 2002; Ackerman et al., 2003; O'Hirok and Gautier, 2003; Oreopoulos et al., 2003]. Based on high-resolution spectral albedo data, along with a state-of-the-art radiative transfer model [Li et al., 2002], [Li et al., 2003] stated that accounting for the heterogeneity of surface albedo could eliminate the systematic difference between measured and modeled cloud absorption. However, the influence of inhomogeneous surface albedo has been ignored in most radiative transfer models. As a result, up to now, there have been no systematic and quantitative analyses of the effects of surface heterogeneity on cloud absorption estimates. This study aims both to understand how more realistic treatments of surface heterogeneity affect cloud absorption and to examine whether the bias between observed and modeled cloud absorption could be explained by inhomogeneous surface albedo.

\section{Approach}

[4] We used the Discrete-Ordinate-method (DISORT) [Stamnes et al., 1988], a Monte Carlo method [Marchuk et al., 1980], and the Spherical Harmonics Discrete Ordinate Method (SHDOM) [Evans, 1998] radiative transfer models to calculate cloud absorption. Models were set up with clouds over a surface with a checkerboard albedo $\alpha$ (shown in Figure 1), where the complexity of clouds increased from homogeneous to broken. The checkerboard surface was changed from the extreme case of black $(\alpha=0)$ and white $(\alpha=1)$, having the largest contrast, to a black and gray $(\alpha=0.5)$ pattern, which is closer to measured albedos for the Atmospheric Radiation Measurement (ARM) program Southern Great Plains (SGP) central facility. Cloud properties are defined via cloud optical depth $\tau$ and single scattering albedo $\varpi_{0}$, and cosine of the solar zenith angle (SZA) is denoted as $\mu_{0}$. For simplicity, molecular scattering, aerosols and gaseous absorption are not taken into account.

[5] Based on energy conservation, cloud absorptance $A$ can be computed from reflectance $R$ and transmittance $T$ as

$$
A(\alpha)=1-R(\alpha)-(1-\alpha) T(\alpha)
$$

where $A, R$, and $T$ are all functions of Lambertian surface albedo $\alpha$, and $(1-\alpha) T$ presents total surface absorption. Note that $A, R$, and $T$ are also functions of $\tau, \varpi_{0}$ and $\mu_{0}$. For plane-parallel clouds,

$$
R(\alpha)=R_{0}+\frac{T_{0}^{2} \alpha}{1-\alpha R^{*}}
$$

and

$$
T(\alpha)=\frac{T_{0}}{1-\alpha R^{*}}
$$

where $R_{0}$ and $T_{0}$ are cloud reflectance and transmittance, respectively, in the case of black surface and $R^{*}$ is the reflectance of clouds when illuminated from below by 


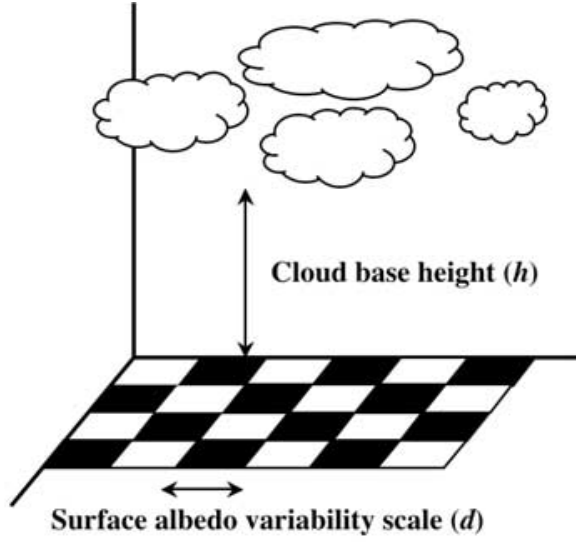

Figure 1. Schematic illustration of model setup.

upwelling isotropic radiation [Liou, 2002, pp. 365-366]. Then, equation (1) can be rewritten as

$$
A(\alpha)=1-R_{0}-\frac{T_{0}\left[\alpha T_{0}+(1-\alpha)\right]}{1-\alpha R^{*}} .
$$

Here $R_{0}, R^{*}$, and $T_{0}$ are independent of surface albedo $\alpha$, and only depend on cloud properties and solar angles.

[6] In order to understand the effects of surface heterogeneity on cloud absorption and to compare the results from both one-dimensional (1D) and three-dimensional (3D) calculations, we used three different approaches. Note that even for plane-parallel 1D clouds, the checkerboard-surface problem requires 3D calculations. The first approach treats the surface with the "surface independent pixel approximation" (SIPA). It computes cloud absorption independently for each column with its associated surface albedo, and then averages absorption over all columns. The second approach, homogeneous-surface (HS), applies an average surface albedo. The third approach involves no approximation and does full 3D radiative transfer calculations. The following sections provide quantitative comparisons of these three approaches for various cases.

\section{Homogeneous Clouds}

\subsection{Cloud Absorption Bias Between SIPA and HS}

[7] Except for the trivial case of $\varpi_{0} \rightarrow 1$ that corresponds to $A \rightarrow 0$, it follows from equation (4) that $d^{2} A / d \alpha^{2} \geq 0$, i.e., $A(\alpha)$ is a concave function. Therefore,

$$
A\left(\frac{\alpha_{1}+\alpha_{2}}{2}\right) \leq \frac{A\left(\alpha_{1}\right)+A\left(\alpha_{2}\right)}{2}
$$

for surface albedos $0 \leq \alpha_{1}, \alpha_{2} \leq 1$. Obviously, the left hand side of equation (5) represents the homogeneous-surface (HS) cloud absorption estimate, while the right hand side corresponds to the SIPA estimate. Therefore, for horizontally homogeneous clouds, averaging surface albedos always decreases cloud absorption.

[8] The above theoretical conclusions can be illustrated by DISORT numerical calculations. Figure 2 depicts the dependency of cloud absorption on surface albedo for one example with overhead sun illumination, an arbitrary optical depth $\tau$ of 16 and various cloud single-scattering albedos

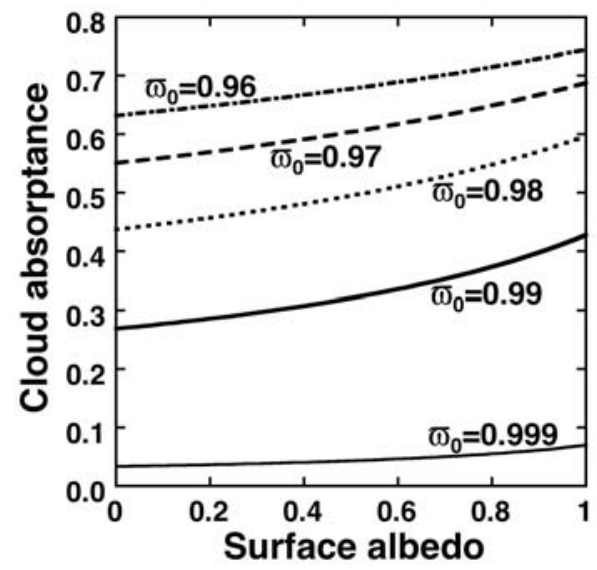

Figure 2. Relationship of cloud absorptance to surface albedo for overhead sun illumination, $\tau=16$, and various cloud single-scattering albedos $\left(\varpi_{0}\right)$. This plot is based on DISORT calculations.

$\varpi_{0}$. Indeed, the cloud absorption follows a concave relationship with surface albedo. For a black $(\alpha=0)$ and white $(\alpha=1)$ checkerboard surface, Figure 3 illustrates the differences between SIPA and HS as a function of $\tau$ for homogeneous clouds, overhead sun, and various $\varpi_{0}$. The bias between SIPA and HS is always positive, which is confirmed by equation (5).

[9] Over all reasonable sets of $\left(\tau, \varpi_{0}, \mu_{0}\right)$ for shortwave radiation and with a black and white checkerboard surface, the biggest absolute difference between SIPA and HS estimates occurs at $\left(\tau, \varpi_{0}, \mu_{0}\right)=(16,0.99,1.0)$ with a value of 0.028 . The difference associated with this particular set of cloud properties and solar angle reflects the most pronounced impact of surface heterogeneity that we produced for homogeneous clouds. It serves as the best situation to examine further how scales of surface heterogeneity affect cloud absorption when $3 \mathrm{D}$ modeling is

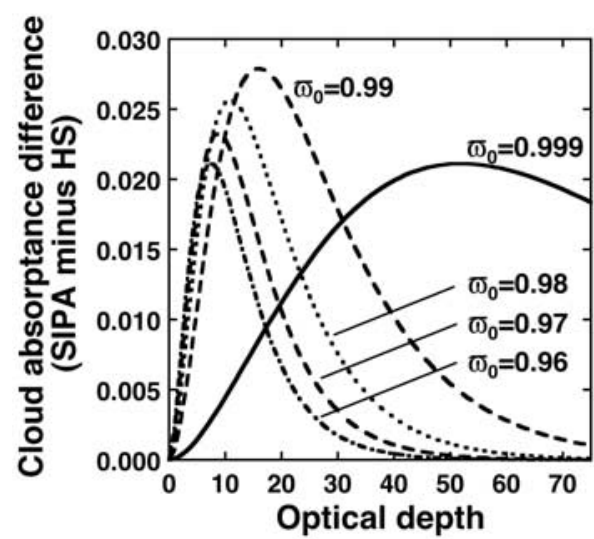

Figure 3. Differences between cloud absorptance estimates from the surface independent pixel approximation (SIPA) and the homogeneous-surface assumption (HS), as a function of cloud optical depth $\tau$. Results are obtained from DISORT assuming a black and white checkerboard surface, overhead sun illumination, and varying single-scattering albedos $\varpi_{0}$ from 0.96 to 0.999 . 


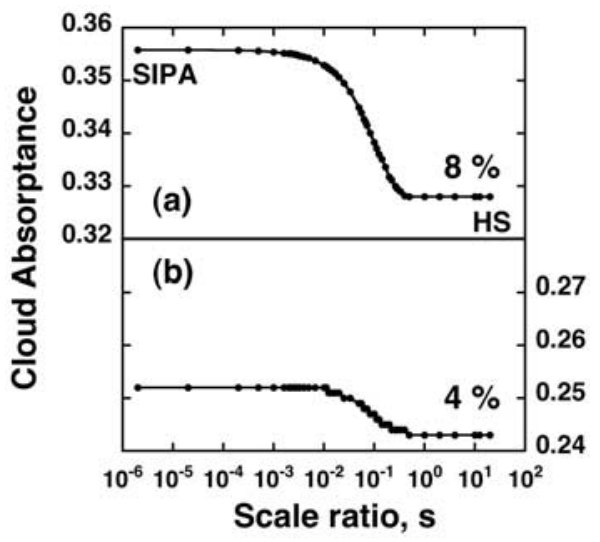

Figure 4. (a) Cloud absorptance as a function of scale ratio with an underlying black and white checkerboard surface for the biggest-effect case: $\left(\tau, \varpi_{0}, \mu_{0}\right)=(16,0.99,1.0)$. In the limiting case of small $s$ cloud absorptance approaches the estimate made by the surface independent pixel assumption. In the limiting case of large $s$ cloud absorptance approaches the homogeneous-surface estimate. (b) Same as (a), but depicting integrations of equation (7) for cloud inhomogeneities, wavelengths, and solar zenith angles. The percentage indicates the relative bias between the smallest and largest scale ratios. This plot is based on Monte-Carlo calculations (SHDOM results are found to be reasonably close).

involved. Thus, for convenience, we refer hereafter to this particular case as the biggest-effect case.

[10] One might argue with the adequacy of the absolute bias between SIPA and HS estimates to determine the biggest-effect case. The biggest absolute difference is of greatest interest here since the effects of surface heterogeneity might be obscured in a case of small absolute but large relative bias due to limitations of model accuracy. Therefore, we define the biggest-effect case based on the absolute difference, although the relative bias will be used as well.

\subsection{D Modeling}

[11] The effects of surface albedo variability on homogeneous clouds from 3D radiative transfer modeling are demonstrated via a scale ratio $s$, defined as

$$
s=\frac{h}{d}
$$

where $h$ is cloud base height and $d$ is the horizontal scale of surface heterogeneity (shown in Figure 1). Figure 4a depicts the curve that relates cloud absorption to the scale ratios for the biggest-effect case. Note that the abscissa is on a logarithmic scale. Cloud absorptance decreases with increasing scale ratio, with a total change of approximately $8 \%$.

[12] In the limiting case of small $s$, due to small $h$ and/or large $d$, photons reflected from a surface pixel will interact only with a cloud located directly above this pixel. In this limit, cloud absorptance approaches the SIPA estimate. In the limiting case of large $s$, cloud absorptance approaches the homogeneous-surface (HS) estimate. For large $s$, cloud absorption in a vertical column is due to photons reflected from many different surface pixels. As a result, the surface looks gray to clouds, which is close to the HS assumption.

\section{Inhomogeneous Clouds}

[13] The previous section has demonstrated the effects of surface heterogeneity on cloud absorption for homogeneous clouds. However, observed clouds are rarely uniform without any internal or horizontal variability [Genkova and Davies, 2003]. Hence, we need to understand whether inhomogeneous surface albedos affect cloud absorption for inhomogeneous clouds in the same manner as they do for homogeneous clouds. To simulate cloud inhomogeneity, a fractionally integrated cascade model [Schertzer and Lovejoy, 1987] was used to generate various cloud structures. For a variety of cloud fractions 50 realizations were produced to include different cloud inhomogeneity. Each realization retains an areal mean optical depth of 16 . These realizations were then used to calculate cloud absorption for $\varpi_{0}=0.99$ and various solar zenith angles and cloud scales. We found that there was no significant qualitative or quantitative difference in the effects of surface heterogeneity for fractal clouds as compared to homogeneous clouds (figures not shown). For 3D clouds, a $5 \sim 7 \%$ change in cloud absorption is found between the two limiting cases of SIPA and HS.

\section{Inclusions of Broadband Spectrum, Diurnal Cycle and Various Clouds}

[14] We have demonstrated how inhomogeneous black and white checkerboard surfaces affect single-wavelength cloud absorption for homogeneous and heterogeneous clouds. When we consider variability in $\tau, \varpi_{0}$, and $\mu_{0}$, the overall cloud absorption $\langle A(\alpha)\rangle$ can be computed as

$$
\begin{aligned}
\langle A(\alpha)\rangle= & \int_{\varpi_{0}} \int_{\mu_{0}} \int_{\tau} A\left(\tau, \varpi_{0}, \mu_{0}, \alpha\right) \\
& \cdot P_{\tau}(\tau) P_{\mu_{0}}\left(\mu_{0}\right) P_{\varpi_{0}}\left(\varpi_{0}\right) d \tau d \mu_{0} d \varpi_{0},
\end{aligned}
$$

where $P_{\tau}, P_{\mu_{0}}$, and $P_{\varpi_{0}}$ represent the probability density functions of $\tau, \mu_{0}$, and $\varpi_{0}$, respectively. $P_{\tau}$ was estimated by the histogram of simulated optical depths from the cascade model in overcast cases. The simulated optical depths were split into five bins. The sample mean of $P_{\tau}$ was $16 . P_{\mu_{0}}$ was approximated by equal weights at zenith angles of 30,45 and $60^{\circ}$. For $P_{\varpi_{0}}$, we divided the solar spectrum into five bands with single scattering albedos of 0.9, 0.98, 0.99, 0.996 , and 1.0. Then, using a simple quadrature rule to solve equation (7), we arrived at an average estimate of the cloud absorption. Note that a more accurate parameterization in probability density functions of equation (7) will not affect our results substantially.

[15] The resulting broadband cloud absorptance over a black and white checkerboard surface is shown in Figure $4 \mathrm{~b}$. The bias due to changes in scale ratio is now less than $4 \%$ after considering cloud inhomogeneity, a diurnal cycle, and broadband absorption. Note that surface albedo $\alpha$ is a function of wavelength and it should not be held constant in the integrations. However, for simplicity black and white surface albedos were employed in the integrations of absorption $A$. This simplification does not weaken our 


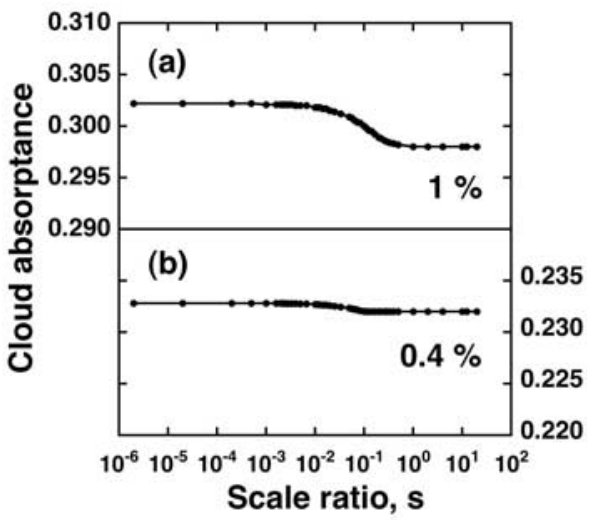

Figure 5. Same as Figure 4, but with a black $(\alpha=0)$ and gray $(\alpha=0.5)$ checkerboard surface in which the albedos are closer to ARM SGP central facility measurements.

demonstration of surface effects on broadband cloud absorption since the bias here is highly exaggerated. This "overestimated" change in cloud absorption showed a substantial reduction from $8 \%$ to $4 \%$ in going from the monochromatic to broadband calculations. One can expect that surface effects on broadband cloud absorption will be even less pronounced due to smaller contrasts in surface albedo.

\section{Black and Gray Checkerboard Surface}

[16] To this point, we have used only a black and white checkerboard surface in the study. One can imagine that this surface pattern describes a water pixel adjacent to an ice pixel, and thus it might not be representative of real-world applications. Since a range of 0.01 to 0.50 in the spectral surface albedo was measured around the ARM SGP central facility [Li et al., 2002; O'Hirok and Gautier, 2003], a black and gray checkerboard with albedo of 0.0 and 0.5 was also tested to understand if surface heterogeneity could explain the observed excess cloud absorption in the ARM SGP observation environment. Results (Figure 5) indicate that the change of cloud absorption is less than $1 \%$ between the smallest and largest scale ratios for both single-wavelength and broadband calculations. This strongly suggests that the discrepancy between observed and modeled cloud absorption cannot be explained by surface heterogeneity.

\section{Conclusions}

[17] This paper presents a systematic and quantitative analysis of the effect of surface heterogeneity on shortwave cloud absorption. We have demonstrated that the use of an averaged surface albedo leads to underestimation of cloud absorption. We also find that in extreme cases, e.g., an underlying black and white checkerboard surface, inhomogeneous surface albedo makes as much as an $8 \%(10-$ $20 \mathrm{~W} / \mathrm{m}^{2}$ ) difference in cloud absorption estimates. However, in reality, the spectral surface albedo around the ARM SGP central facility is around $0.01-0.50$. In that observation environment, for any situation of clouds and solar illumination, the effect of surface heterogeneity on cloud absorption is negligible, showing less than $0.5 \%$, or $\sim 1 \mathrm{~W} / \mathrm{m}^{2}$. This $1 \mathrm{~W} / \mathrm{m}^{2}$ difference attributed to surface albedo is not only less than the uncertainty caused from other variables, such as water vapor and aerosols, and errors in radiative transfer models themselves, but also much less than the discrepancy (order of $10 \mathrm{~W} / \mathrm{m}^{2}$ ) between measured and model-calculated cloud absorption. Therefore, this study strongly indicates that accounting for surface heterogeneity in radiative transfer models cannot explain anomalous shortwave cloud absorption.

[18] Acknowledgment. This research was supported by the Office of Science (BER, U.S. Department of Energy, Interagency Agreement No. DE-AI02-95ER61961) as part of the ARM program.

\section{References}

Ackerman, T. P., D. M. Flynn, and R. T. Marchand (2003), Quantifying the magnitude of anomalous solar absorption, J. Geophys. Res., 108(D9), 4268, doi:10.1029/2002JD003000.

Barker, H. W. (1992), Solar radiative transfer through clouds possessing isotropic variable extinction coefficient, Q. J. R. Meteorol. Soc., 118, $1145-1162$

Cess, R. D., M. Zhang, F. P. J. Valero, A. S. Simpson, and L. Bignone (1999), Absorption of solar radiation by the cloudy atmosphere: Further interpretations of collocated aircraft measurements, J. Geophys. Res., $104,2059-2066$.

Evans, K. F. (1998), The spherical harmonics discrete ordinate method for three-dimensional atmospheric radiative transfer, J. Atmos. Sci., 55, 429446.

Genkova, I., and R. Davies (2003), Spatial heterogeneity of reflected radiance from globally distributed clouds, Geophys. Res. Lett., 30(21), 2096, doi:10.1029/2003GL018194.

Knyazikhin, Y., A. Marshak, W. J. Wiscombe, J. V. Martonchik, and R. B. Myneni (2002), A missing solution to the transport equation and its effect on estimation of cloud absorptive properties, J. Atmos. Sci., 59, $3572-$ 3585 .

Li, Z., M. C. Cribb, and A. P. Trishchenko (2002), Impact of surface inhomogeneity on solar radiative transfer under overcast conditions, J. Geophys. Res., 107(D16), 4294, doi:10.1029/2001JD000976.

Li, Z., T. Ackerman, W. Wiscombe, and G. L. Stephens (2003), Have clouds darkened since 1995?, Science, 302(5648), 1151, doi:10.1126/ science.302.5648.1151.

Liou, K. N. (Ed.) (2002), An Introduction to Atmospheric Radiation, 583 pp., Academic, San Diego, California.

Marchuk, G., G. Mikhailov, M. Nazaraliev, R. Darbinjan, B. Kargin, and B. Elepov (Eds.) (1980), The Monte Carlo Methods in Atmospheric Optics, 208 pp., Springer-Verlag, New York.

Marshak, A., A. Davis, W. Wiscombe, and R. Cahalan (1997), Inhomogeneity effects on cloud shortwave absorption measurements: Two-aircraft simulations, J. Geophys. Res., 102, 16,619-16,637.

O'Hirok, W., and C. Gautier (2003), Absorption of shortwave radiation in a cloudy atmosphere: Observed and theoretical estimates during the second Atmospheric Radiation Measurement Enhanced Shortwave Experiment (ARESE), J. Geophys. Res., 108(D14), 4412, doi:10.1029/ 2002JD002818.

Oreopoulos, L., A. Marshak, and R. F. Cahalan (2003), Consistency of ARESE II cloud absorption estimates and sampling issues, J. Geophys. Res., 108(D1), 4029, doi:10.1029/2002JD002243.

Schertzer, D., and S. Lovejoy (1987), Physical modeling and analysis of rain and clouds by anisotropic scaling multiplicative processes, J. Geophys. Res., 92, 9693-9714.

Stamnes, K., S.-C. Tsay, W. Wiscombe, and K. Jayaweera (1988), Numerically stable algorithm for discrete-ordinate-method radiative transfer in multiple scattering and emitting layered media, Appl. Opt., 27, 25022512.

Valero, F. P. J., R. D. Cess, M. Zhang, S. K. Pope, A. Bucholtz, B. Bush, and J. Vitko Jr. (1997), Absorption of solar radiation by clouds: Interpretations of collocated aircraft measurements, J. Geophys. Res., 102, 29,917-29,927.

Valero, F. P. J., S. K. Pope, B. C. Bush, Q. Nguyen, D. Marsden, R. D. Cess, A. S. Simpson-Leitner, A. Bucholtz, and P. M. Udelhofen (2003), Absorption of solar radiation by the clear and cloudy atmosphere during the Atmospheric Radiation Measurement Enhanced Shortwave Experiments (ARESE) I and II: Observations and models, J. Geophys. Res., 108(D1), 4016, doi:10.1029/2001JD001384.

J.-Y. C. Chiu, JCET/UMBC, 1000 Hilltop Circle, Baltimore, MD 21250, USA. (cchiu@climate.gsfc.nasa.gov)

A. Marshak and W. J. Wiscombe, Climate and Radiation Branch, NASA Goddard Space Flight Center, Greenbelt, MD, USA. 